\title{
COMMON FIXED POINT THEOREMS FOR COMMUTING MAPS ON A METRIC SPACE
}

\author{
K. M. DAS AND K. VISWANATHA NAIK
}

ABSTRACT. Results generalizing and unifying fixed point theorems of Jungck and Ciric are established.

1. The purpose of this paper is to generalize and unify fixed point theorems of Jungck [2] and Ciric [1] (cf. Theorems 1.1 and 1.2 below). As a corollary of the main result (Theorem 3.1) another result (Theorem 3.2) concerning commuting, not necessarily continuous, mappings is also proved.

Theorem 1.1 (G. JungCK [2]). Let $X$ be a complete metric space. Let $f$ and $g$ be commuting continuous self-maps on $X$ such that

$$
g(X) \subset f(X) \text {. }
$$

Further, let there exist a constant $\alpha \in(0,1)$ such that for every $x, y$ in $X$

$$
d(g x, g y) \leqslant \alpha d(f x, f y) .
$$

Then $f$ and $g$ have a unique common fixed point.

TheOREM 1.2 (CIRIC [1]). Let $X$ be a complete metric space. Let $f$ be a self-map on $X$ such that for some constant $\alpha \in(0,1)$ and for every $x, y$ in $X$

$$
d(f x, f y) \leqslant \alpha \max \{d(x, y), d(x, f x), d(y, f y), d(x, f y), d(y, f x)\} .
$$

Then $f$ possesses a unique fixed point.

We remark that (1.3) is the most general among contractive type conditions studied by various authors. For details about these refer to Rhoades [3].

2. In this section, we prove a fixed point theorem which is a generalization of Theorem 1.1.

Let $X$ be a complete metric space. Let $f$ be a continuous self-map on $X$ and let $g$ be any self-map on $X$ such that (1.1) is satisfied. We define a sequence of points $\left\{x_{n}\right\}$ as follows. For $x_{0}(\in X)$ arbitrary, let $x_{1}(\in X)$, guaranteed by (1.1), be such that $g\left(x_{0}\right)=f\left(x_{1}\right)$. Having defined $x_{n}(\in X)$, let $x_{n+1}(\in X)$ be such that $g\left(x_{n}\right)=f\left(x_{n+1}\right)$.

Letting $g\left(x_{n}\right)\left(=f\left(x_{n+1}\right)\right)=y_{n}(n=0,1,2, \ldots)$ we denote by $O\left(y_{k} ; n\right)$ the set of points $\left\{y_{k}, y_{k+1}, \ldots, y_{k+n}\right\}$. Let us assume that $f$ and $g$ satisfy the following condition:

There exists a constant $\alpha \in(0,1)$ such that for every $x, y$ in $X$,

Received by the editors September 1, 1978.

AMS (MOS) subject classifications (1970). Primary 47H10; Secondary 54H25.

(c) 1979 American Mathematical Society $0002-9939 / 79 / 0000-0564 / \$ 02.25$ 


$$
\begin{array}{r}
d(g x, g y)<\alpha \max \{d(f x, f y), d(f x, g x), d(f y, g y), \\
d(f x, g y), d(f y, g x)\} .
\end{array}
$$

We begin with some results about $\delta\left(O\left(y_{k} ; n\right)\right)$, the diameter of $O\left(y_{k} ; n\right)$, in the form of lemmas. (These are slight variations on the ones in Ciric [1].) The proofs are indicated for the sake of completeness.

LEMMA 2.1. For $k \geqslant 0$ and $n \in N$, suppose $\delta\left(O\left(y_{k} ; n\right)\right)>0$. Then $\delta\left(O\left(y_{k} ; n\right)\right)=d\left(y_{k}, y_{j}\right)$, where $j$ is such that $k<j \leqslant k+n$. Also

$$
\delta\left(O\left(y_{k} ; n\right)\right) \leqslant \alpha \delta\left(O\left(y_{k-1} ; n+1\right)\right) \quad(k \geqslant 1) .
$$

Proof. For $i, j$ such that $1 \leqslant i<j$,

$$
\begin{aligned}
d\left(y_{i}, y_{j}\right) & =d\left(g x_{i}, g x_{j}\right) \\
< & \alpha \max \left\{d\left(f x_{i}, f x_{j}\right), d\left(f x_{i}, g x_{i}\right), d\left(f x_{j}, g x_{j}\right), d\left(f x_{i}, g x_{j}\right), d\left(f x_{j}, g x_{i}\right)\right\} \\
= & \alpha \max \left\{d\left(y_{i-1}, y_{j-1}\right), d\left(y_{i-1}, y_{i}\right), d\left(y_{j-1}, y_{j}\right), d\left(y_{i-1}, y_{j}\right), d\left(y_{j-1}, y_{i}\right)\right\} .
\end{aligned}
$$

Thus

$$
d\left(y_{i}, y_{j}\right)<\alpha \delta\left(O\left(y_{i-1} ; j-i+1\right)\right) .
$$

Now $\delta\left(O\left(y_{k} ; n\right)\right)=d\left(y_{i}, y_{j}\right)$, for some $i, j$ satisfying $k<i<j<k+n$, in view of the fact that the supremum of a finite number of distances is taken.

If $i>k$, then by (2.3):

$$
\delta\left(O\left(y_{k} ; n\right)\right)<\alpha \delta\left(O\left(y_{i-1} ; j-i+1\right)\right)
$$

with $i-1>k$ and $j<k+n$, whence

$$
\delta\left(O\left(y_{k} ; n\right)\right) \leqslant \alpha \delta\left(O\left(y_{k} ; n\right)\right),
$$

a contradiction. This proves the first assertion. Moreover,

$$
\begin{aligned}
\delta\left(O\left(y_{k} ; n\right)\right) & =d\left(y_{k}, y_{j}\right)<\alpha \delta\left(O\left(y_{k-1} ; j-k+1\right)\right) \\
& <\alpha \delta\left(O\left(y_{k-1} ; n+1\right)\right) .
\end{aligned}
$$

LEMMA 2.2. Under the hypotheses of Lemma 2.1,

$$
\delta\left(O\left(y_{k} ; n\right)\right) \leqslant \frac{\alpha^{k}}{1-\alpha} d\left(y_{0}, y_{1}\right)
$$

Proof.

$$
\begin{aligned}
\delta\left(O\left(y_{l} ; m\right)\right) & =d\left(y_{l}, y_{j}\right)<d\left(y_{l}, y_{1+l}\right)+d\left(y_{1+l}, y_{j}\right) \\
& <d\left(y_{l}, y_{1+l}\right)+\delta\left(O\left(y_{1+l} ; m-1\right)\right),
\end{aligned}
$$

since $j<l+m$. Thus

$$
\delta\left(O\left(y_{l} ; m\right)\right)<d\left(y_{l}, y_{1+l}\right)+\alpha \delta\left(O\left(y_{l} ; m\right)\right),
$$

in view of (2.3). This leads to

$$
\delta\left(O\left(y_{l} ; m\right)\right)<\frac{1}{1-\alpha} d\left(y_{l}, y_{1+l}\right)
$$


By repeated application of (2.2) we have

$$
\delta\left(O\left(y_{k} ; n\right)\right)<\alpha^{k} \delta\left(O\left(y_{0} ; n+k\right)\right),
$$

whence (2.4) follows in view of (2.5) with $l=0, m=n+k$.

THEOREM 2.1. Let $X$ be a complete metric space. Let $f$ be a continuous self-map on $X$ and $g$ be any self-map on $X$ that commutes with $f$. Further, let $f$ and $g$ satisfy (1.1) and (2.1). Then $f$ and $g$ have a unique common fixed point.

Proof. We first remark that it is sufficient to produce a point $y$ such that $f(y)=g(y)$. For then

$$
\begin{aligned}
d(g g y, g y) & <\alpha \max \{d(f g y, f y), d(f g y, g g y), d(f y, g y), d(f g y, g y), d(f y, g g y)\} \\
& =\alpha d(g g y, g y)
\end{aligned}
$$

shows that $g(y)$ is a fixed point of $g$. Observing $f(g y)=g(f y)=g(g y)=$ $g(y)$, we see that $g(y)$ is also a fixed point of $f$.

If for some $n$ and $k, \delta\left(O\left(y_{k} ; n\right)\right)=0$ we have $y_{k}=y_{k+1}$, i.e., $f\left(x_{k+1}\right)=$ $g\left(x_{k+1}\right)$. Otherwise $\delta\left(O\left(y_{k} ; n\right)\right)>0$. Given $\varepsilon>0$, let $n_{0} \in N$ be such that $\alpha^{n} d\left(y_{0}, y_{1}\right)<(1-\alpha) \varepsilon$. Thus for $m>n \geqslant n_{0}$,

$$
d\left(y_{m}, y_{n}\right) \leqslant \delta\left(O\left(y_{n_{0}} ; m-n_{0}\right)\right)<\varepsilon
$$

in view of Lemma 2.2 and the choice of $n_{0}$.

Hence $\left\{y_{n}\right\}$, a Cauchy sequence in a complete metric space, has a limit, say $y$. By continuity of $f,\left\{f\left(y_{n}\right)\right\}$ converges to $f(y)$. Moreover, $\left\{g\left(y_{n}\right)(=\right.$ $\left.f\left(y_{n+1}\right)\right)$ \} also converges to $f(y)$. Further,

$$
\begin{aligned}
d\left(f y_{n+1}, g y\right) & =d\left(g y_{n}, g y\right) \\
< & \alpha \max \left\{d\left(f y_{n}, f y\right), d\left(f y_{n}, g y_{n}\right), d(f y, g y), d\left(f y_{n}, g y\right), d\left(f y_{n}, g y_{n}\right)\right\}
\end{aligned}
$$

in the limit leads to $d(f y, g y)<\alpha d(f y, g y)$, whence $f y=g y$ for otherwise $\alpha>1$.

The uniqueness of the common fixed point is immediate from (2.1).

REMARK 1. In Theorem 2.1 , setting $f I_{X}$, the identity map on $X$, we get Theorem 1.2. Moreover, Theorem 1.1 follows from Theorem 2.1 on noting that (1.2) implies (2.1).

REMARK 2. If in place of $g$ some iterate $g^{i}$ of $g$ satisfies the hypotheses (1.1) and (2.1), then also the conclusion of Theorem 2.1 follows in view of $f(g(\bar{x}))=g^{i}(g(\bar{x}))=g(\bar{x})$, where $\bar{x}$ is the unique common fixed point of $f$ and $g^{i}$.

REMarK 3. If $\left\{g_{\lambda}\right\}_{\lambda \in \Lambda}$ is a commuting family of self-maps on $X$ such that for each $\lambda \in \Lambda$, the hypotheses of Theorem 2.1 hold with $g$ replaced by $g_{\lambda}$, then it is easy to see that there exists a unique point $\bar{x}$ such that $f(\bar{x})=g_{\lambda}(\bar{x})$ $=\bar{x}$ for every $\lambda \in \Lambda$.

3. In the next theorem the domain of $g$ coincides with $f(X)$. Also the weaker hypothesis, namely, continuity of $f^{2}$ (in place of that of $f$ ) suffices. We conclude the section with an example to show that the conclusion of Jungck's 
theorem holds for $f$ not necessarily continuous.

THEOREM 3.1. Let $X$ be a complete metric space. Let $f$ be a self-map on $X$ such that $f^{2}$ is continuous. Let $g: f(X) \rightarrow X$ be such that

$$
g f(X) \subset f^{2}(X)
$$

and $f(g(x))=g(f(x))$ whenever both sides are defined. Further, let there exist a number $\alpha \in(0,1)$ such that $(2.1)$ holds for every $x, y$ in $f(X)$. Then $f$ and $g$ have a unique common fixed point.

Proof. Starting with an arbitrary point $x_{0}$ in $f(X)$ and appealing to condition (3.1), we construct a sequence $\left\{x_{n}\right\}$ of points in $f(X)$ such that $f\left(x_{n+1}\right)=g\left(x_{n}\right)=y_{n}$, say. Note that $f\left(y_{n}\right)=f\left(g\left(x_{n}\right)\right)=g\left(f\left(x_{n}\right)\right)=g\left(y_{n-1}\right)=$ $z_{n}$, say.

Arguing as in Lemmas 2.1 and 2.2, we get that for $k \geqslant 0, n \in N$,

$$
\delta\left(O\left(z_{k} ; n\right)\right) \leqslant \frac{\alpha^{k}}{1-\alpha} d\left(z_{0}, z_{1}\right) .
$$

This yields that $\left\{z_{n}\right\}$ is a Cauchy sequence in $X$ and hence convergent to some $z$ in $X$. By continuity of $f^{2},\left\{f^{2}\left(z_{n}\right)\right\}$ converges to $f^{2}(z)$.

Moreover, $g f\left(z_{n}\right)=g f\left(f^{2} x_{n+1}\right)=f^{2}\left(f\left(g x_{n+1}\right)\right)=f^{2}\left(z_{n+1}\right)$ implies that $\left\{g f\left(z_{n}\right)\right\}$ converges to $f^{2}(z)$. Further,

$$
\begin{aligned}
& d\left(f^{2} z_{n+1}, g f z\right)= d\left(g f z_{n}, g f z\right) \\
& \leqslant \alpha \max \left\{d\left(f^{2} z_{n}, f^{2} z\right), d\left(f^{2} z_{n}, g f z_{n}\right),\right. \\
&\left.d\left(f^{2} z, g f z\right), d\left(f^{2} z_{n}, g f z\right), d\left(f^{2} z, g f z_{n}\right)\right\}
\end{aligned}
$$

in the limit leads to

$$
d\left(f^{2} z, g f z\right) \leqslant \alpha d\left(f^{2} z, g f z\right) .
$$

Thus $f^{2} z=g f z$.

Finally,

$$
\begin{aligned}
d(g(g f z), g f z) \leqslant & \alpha \max \left\{d\left(f g f z, f^{2} z\right), d(f g f z, g g f z),\right. \\
& \left.d\left(f^{2} z, g f z\right), d(f g f z, g f z), d\left(f^{2} z, g g f z\right)\right\} \\
& =\alpha d(g(g f z), g f z)
\end{aligned}
$$

yields $g(g f z)=g f z ; g f z$ can be easily seen to be a fixed point of $f$ also. Hence $f$ and $g$ have a common fixed point. The uniqueness follows once again from (2.1).

As an easy corollary of this we have:

TheOrem 3.2. Assume that all the hypotheses of Theorem 1.1 except continuity of $f$ hold. Let $f^{2}$ be continuous. Then $f$ and $g$ have a unique common fixed point. 
Proof. Note that $f(X) \supset g(X)$ implies that $f^{2}(X) \supset f(g(X)=g(f(X)))$, i.e., (3.1) holds. Since (2.1) obviously holds, the conclusion follows in view of Theorem 3.1.

The following example illustrates that Theorem 3.2 is indeed a generalization of Jungck's theorem.

Example. Let $X$ be $(-\infty, \infty)$ with the usual metric. Define $f: X \rightarrow X$ as follows

$$
f(x)= \begin{cases}1 & \text { for } x<-1, \\ x & \text { for }-1<x<1 \\ -1 & \text { for } x \geqslant 1\end{cases}
$$

Let $g$ be defined over $X$ by

$$
g(x)= \begin{cases}\frac{1+x}{2} & \text { for }-1 \leqslant x \leqslant 0 \\ \frac{1-x}{2} & \text { for } 0<x \leqslant 1 \\ 0 \text { for } x \in(-\infty,-1] \cup[1, \infty)\end{cases}
$$

Clearly, $f$ is discontinuous, but $f^{2}$ is continuous. It is easy to verify that all the other hypotheses of Theorem 1.1 hold. The (unique) common fixed point, namely $1 / 3$, is guaranteed by Theorem 3.2. It is obvious that Jungck's theorem, however, is not applicable.

4. It is almost evident from the proof of Theorem 3.1 that the following generalization is valid. Since the proof is basically the same, we only state the result.

THEOREM 4.1. Let $X$ be a complete metric space, $f$ a self-map on $X$ such that $f^{m}, m$ any fixed positive integer, is continuous. Let $g: f^{m-1}(X) \rightarrow X$ be such that

$$
g\left(f^{m-1}(X)\right) \subset f^{m}(X)
$$

and $g$ and $f$ commute. Further let there exist a number $\alpha \in(0,1)$ such that $(2.1)$ is satisfied for every $x, y$ in $f^{m-1}(X)$. Then $f$ and $g$ have a unique common fixed point.

\section{REFERENCES}

1. Lj. B. Ciric, A generalization of Banach's contraction principle, Proc. Amer. Math. Soc. 45 (1974), 267-273. MR 50 \#8484.

2. Gerald Jungck, Commuting mappings and fixed points, Amer. Math. Monthly 83 (1976), 261-263. MR 53 \# 4031.

3. B. E. Rhoades, A comparison of various definitions of contractive mappings, Trans. Amer. Math. Soc. 226 (1977), 256-290.

Department of Mathematics, Indian Institute of Technology, Madras 600036, India 